$\mathrm{ml}$ in der Salmeterol-Kombinationsgruppe und um $80 \mathrm{ml}$ in der Tiotropium-Kombinationsgruppe jeweils im Vergleich zu Placebo (beides $p<0,0001$ ).

Das belegt laut Magnussen klar eine Extrawirkung über die gute Wirksamkeit der bisher eingesetzten Substanzen hinaus. Die Untersucher beobachteten auch eine Verbesserung anderer Lungenfunktionsparameter, und die Patienten berichteten von einer spürbaren Erleichterung. Übelkeit, Durchfall und Gewichtsverlust waren die häufigsten Nebenwirkungen. Der Gewichtsverlust lag aber laut Magnussen in den Studien im Mittel nur bei $2 \mathrm{~kg}$ in 48 Monaten und betraf vorrangig die übergewichtigen Patienten.

Magnussen hält Roflumilast für so erfolgversprechend, dass es seiner Ansicht nach nicht lange bei der Einschränkung der Zulassung für den Einsatz bei schwerer und sehr schwerer COPD mit starkem Husten/Auswurf und häufigen Exazerbationen bleiben wird.

- Friederike Klein

Quelle: Pressekonferenz zur Einführung von Roflumilast, Oranienburg, 1. September 2010 (Veranstalter: Nycomed $\mathrm{GmbH}$ )

Typ-2-Diabetes

\title{
Gefäßschutz durch GLP-1-Mimetikum?
}

- Typ-2-Diabetiker zählen zu den hochgradig gefährdeten Gefäßpatienten. GLP-1-Rezeptor-Agonisten können ihnen möglicherweise kardiovaskulären Schutz liefern, so Prof. Petra-Maria SchummDraeger, München.

So kann Exenatide (Byetta ${ }^{\circledR}$ ) gleich mit mehreren Vorteilen für die Patienten aufwarten. Neben den günstigen Effekten auf $\mathrm{HbA}_{1 \mathrm{c}}$-Wert und Gewicht, begleitet von einem geringen Unterzuckerungsrisiko, bietet es auch noch einen kardiovaskulären Zusatznutzen. Dafür sprechen jedenfalls Daten einer Langzeit-Untersuchung, die Prof. Thomas Forst aus Mainz präsentierte. Sie umfasst 151 Typ-2-Diabetiker mit einem mittleren $\mathrm{HbA}_{1 \mathrm{c}}$-Ausgangswert von $8,2 \%$ und einem Gewicht von 99 kg.

Die Patienten wurden drei Jahre lang mit Exenatide behandelt. Dabei zeigte sich zunächst die schnelle Wirkung auf den $\mathrm{HbA}_{1 \mathrm{c}}$, der sich innerhalb von 26 Wochen auf rund $7 \%$ verbesserte. Dieser Ef- fekt war stabil: In den folgenden 2,5 Jahren änderte sich die Einstellungsqualität der Studienteilnehmer nur noch wenig.

\section{Positive Auswirkungen auf Blutdruck und Blutfette}

Gleichzeitig sank der Blutdruck um 3,5/3,3 $\mathrm{mmHg}$. Außerdem wurde am Ende der Studie eine signifikante Zunahme des HDL-Cholesterins um $24 \%$ registriert, während LDL-Cholesterin und Triglyzeride signifikant um $6 \%$ und $12 \%$ abgenommen hatten. Das Gewicht der Patienten ging im Lauf der drei Jahre kontinuierlich zurück, durchschnittlich um 5,3 kg.

Darüber hinaus gibt es erste Hinweise auf mögliche endotheliale Effekte aus einer weiteren Arbeit. Sie ermittelte unter Exenatide eine postprandial verbesserte Pulselastizität in der Aorta.

- Helga Brettschneider

Quelle: Symposium, DDG-Tagung, Stuttgart,

13. Mai 2010 (Veranstalter: Lilly Deutschland)

\section{Depression}

\section{Effektive Schmerzlinderung erhöht die Aussicht auf Remission}

- Im Management von Depressionen steht der Arzt drei Problemen gegenüber: (1) Ein Großteil der Patienten berichtet nur über körperlich-schmerzhafte Beschwerden, sodass eine zugrundeliegende Depression leicht verkannt wird. Die körperlichen Beschwerden müssen sorgfältig abgeklärt werden. Wenn organische Ursachen ausgeschlossen werden können, sei eine Depression wahrscheinlich, so Dr. Armin Spingler, Blaustein.

(2) Die Übermittlung der Diagnose „Depression“ kann sich als schwierig erweisen und erfordert ein Höchstmaß an Empathie.

(3) Die Therapieadhärenz ist oft gering.

Als wirksame Maßnahme empfiehlt Dr. Michael Lang, Ulm, u.a. Patientenschulungen. Mit der Patientenakademie NeuroPoint (www.neuropoint.de) hat der

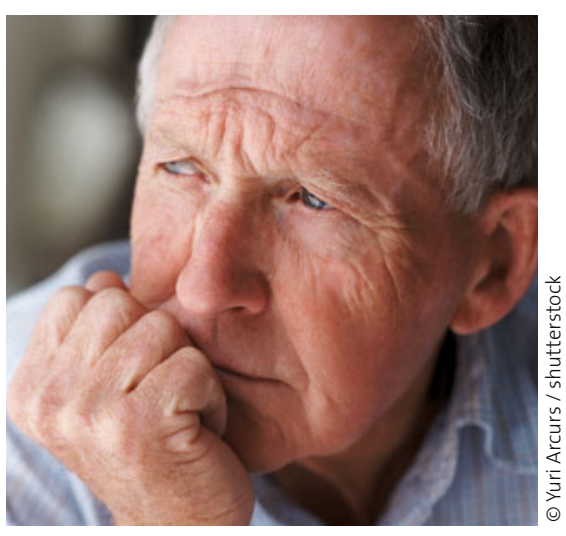

Schmerzen können eine Depression nicht nur maskieren, sondern auch prolongieren.

Neurologe vor 15 Jahren ein Konzept für krankheitsspezifische Patientenseminare initiiert. Das bessere Verständnis für Krankheitsgeschehen und Therapie sei mit höheren Adhärenzraten verknüpft.
Duloxetin (Cymbalta ${ }^{\circledR}$ ) verbessert beide Symptomkomplexe der Depression. Die Substanz bewirkt eine relativ ausgewogene Wiederaufnahmehemmung von Serotonin und Noradrenalin und hat eine direkte Wirkung auf psychische und körperlich-schmerzhafte Beschwerden der Depression. Im Vergleich zu SSRI (Paroxetin bzw. Fluoxetin $20 \mathrm{mg} / \mathrm{d}$ oder Escitalopram $10 \mathrm{mg} / \mathrm{d}$ ) zeigte eine Post-hoc-Analyse für Duloxetin eine signifikant stärkere Besserung des $\mathrm{HAMD}_{17}$-Scores. Im Hinblick auf die Remission ist eine frühe Schmerzlinderung entscheidend. In der PADRE-Studie war eine frühzeitige Schmerzreduktion um $\geq 50 \%$ mit einer höheren Remissionsrate assoziiert (66,9 vs. $36,5 \%$ ).

- Abdol A. Ameri

Quelle: Pressegespräch, Ulm, 28. September 2010 (Veranstalter: Lilly) 